\title{
Improvement of interaction in and properties of PMMA-MWNT nanocomposites through microwave assisted acid treatment of MWNT
}

\author{
A. Zanotto ${ }^{\mathrm{a}, *}$, A.S. $_{\text {Luyt }}{ }^{\mathrm{b}}$, A. Spinella ${ }^{\mathrm{a}}$, E. Caponetti ${ }^{\mathrm{a}, \mathrm{c}}$ \\ ${ }^{a}$ Centro Grandi Apparecchiature-UniNetLab, University of Palermo, Via F. Marini 14, Palermo I-90128, Italy \\ ${ }^{\mathrm{b}}$ Department of Chemistry, University of the Free State (Qwaqwa Campus), Private Bag X13, Phuthaditjhaba 9866, South Africa \\ 'Department of Chemistry "S.Cannizzaro", University of Palermo, Parco d'Orleans II-Viale delle Scienze pad.17, Palermo I-90128, Italy
}

\section{A R T I C L E I N F O}

\section{Article history:}

Received 22 May 2012

Received in revised form 17 October 2012

Accepted 28 October 2012

Available online 22 November 2012

\section{Keywords:}

PMMA

Multi-walled carbon nanotubes

Microwave-assisted purification

Nanocomposite structure

Thermal properties

\begin{abstract}
A B S T R A C T
Soluble derivatives of multi-walled carbon nanotubes (MWNT) embedded in a poly(methylmethacrylate) (PMMA) matrix forming thick, homogeneous and transparent nanocomposites, were prepared and characterized. A new photo-assisted method using microwaves, to purify the MWNTs from amorphous carbon and synthesis catalyst clusters, was tested in a sulphonitric mix. This method shortened the processing time compared to other methods. Pristine and functionalized MWNTs were introduced into the MMA, then in situ photo-polymerized. Transmission electron microscopy (TEM) and X-ray diffractometry (XRD), as well as Fourier-transform infrared (FTIR), Raman and nuclear magnetic resonance (NMR) spectroscopy were used to monitor the effects of the treatment on the different components. The thermal properties of the composites were determined through differential scanning calorimetry (DSC) and thermogravimetric analysis (TGA). The short, microwave treated and polyethylene glycol (PEG) functionalized MWNTs showed the best dispersion in and interaction with PMMA, and had the most significant influence on the thermal properties of this polymer.
\end{abstract}

(c) 2012 Elsevier Ltd. All rights reserved.

\section{Introduction}

Carbon nanotubes (CNT) are materials that show remarkable optoelectronic and physico-mechanical features [1]. CNT is nowadays frequently used in composites in the search for new interesting materials. Multi-walled carbon nanotubes (MWCNT) are obtained by a chemical vapor deposition (CVD) method. The cluster stays inside the nanotube and the synthesis also produces an indefinite amount of amorphous carbon and graphite, which influence the MWCNTs' properties. Purification is necessary to take the best advantage of this innovative material [2].

In literature there are many ways to purify carbon nanotubes, among the more effective ones are thermal [3], acid-oxidative photo-assisted purification [4] and

\footnotetext{
* Corresponding author.

E-mail address: zanotto.antonio@gmail.com (A. Zanotto).
}

refluxing with a strong acid [5]. The method described below allows the oxidation by fullerenic cap cutting, amorphous carbon and the removal of metallic clusters. The purification occurs by means of oxidative-acid attack, photo-assisted by microwaves.

Despite the lack of an identical set of conditions, comparison of acid reflux and microwave assisted acid digestion suggests that the ultrasound and microwave approaches are better. The combined action of the ultrasound and the sulphonitric mix causes breakdown of the bundles and breaking of the fullerenic cup. The latter is the most reactive part because of its curvature and, therefore, they are immediately removed and degraded. The reaction mechanism consists of forming the nitronioum ion that, assisted by ultrasound, reacts with the defects on the walls and with the most bent zones, provoking the shortening of tubes. Furthermore, ultrasound helps with exfoliation, avoiding the degradation of external tubes in the big bundles. The process is unfortunately very 
slow with MWNT, so different microwave photo-assisted cutting methods were studied to accelerate it.

The reaction rate improves under microwave heating due to mixing effects. The rapid molecular rotation at the solvent/solute boundary layer, induced by the electric field, provides efficient localized mixing, in addition to the refluxing effect. A comparison between traditional acid reflux and microwave assisted purification undertaken at similar bulk solution temperatures, demonstrates that microwave assisted purification is significantly more efficient. The efficiency of microwave purification is likely due to the microwave specific phenomenon of super-heating. Dipolar polarization is the principal heat-generating pathway for solvents used in microwave assisted acid digestion. The rapid molecular rotation induced by electric field oscillation is responsible for the heating and mixing benefits. In addition to dipolar polarization, the presence of electrical conductors in the purification system (carbon, metals, solvent ions) means the effect of conductive heating is potentially non-trivial. In this mechanism the microwaves do not directly heat the conducting materials, but the electrons are rather induced to move in the electric field, causing sample heating due to electrical resistance. This localized super-heating at the site of impurities may be responsible for the purification rate increases.

CNTs produced via the CVD method will be contaminated with nanometer scale catalyst metal impurities at the tips, or within the structure of the CNT, which must be removed for high purity products. Therefore, heating induced in the metal catalyst particles generates localized super-heating at the exact site of the impurities, which serves to increase dissolution. However, the behavior of nanometer scale metals under microwave radiation remains unclear. Wadhawan et al. [6] presented a TEM image of iron nanoparticles liquefying and coalescing into large crystallites approximately four times larger than the original nanoparticles during CNT's post microwave treatment at $1000 \mathrm{~W}$ and $2.45 \mathrm{GHz}$. This difference in temperature was attributed to local super-heating of these iron nanoparticles. Imholt et al. [7] found no difference in the thermal behavior of purified and non-purified CNTs in a microwave field.

A 'perfect' CNT could be a ballistic conductor, and its own resistance is quantized and depends on its length, due to its unique 1D structure. This means that no energy would be dissipated in the CNT due to electron movement, allowing extremely high stable current densities, without structural damage [8]. However, as CVD synthesized CNT products typically have structural imperfections, the ballistic transportation properties decay, allowing joule heating to occur in the CNT, leading to surface super-heating. This has the potential benefit that the defective and damaged CNTs may be supplied with sufficient energy to reorient any 'imperfect' sp3 carbon bonds into the desired sp2 configuration, or that the defective CNTs are thermally destroyed leading to an increase in average CNT quality at the expense of purification yield. This behavior may vary slightly between different CNTs due to differing chiral angles leading to slight variations in temperature and hence purification efficiency for metallic or semiconducting CNTs, as speculated by Chen et al. [9].
Microwave radiation may also give rise to mixing enhancements. This has the effect that the surface of the CNTs very efficiently attenuates the electromagnetic waves and thus shields the majority of the as-synthesized CNT samples from the energy source. Depending on the microwave frequency, the small penetration depth may require very thin layers of CNTs or bulk circulation for efficient large-scale continuous processing.

The purification by means of oxidative-acid attack allows one to obtain purified MWNT, lacking in fullerenic caps, with oxidized extremities for further functionalization. Thin transparent and homogeneous films of pristine NT composites have already been synthesized [10], but a soluble derivative is necessary to guarantee transparency and homogeneity in thick and concentrated samples.

In this paper, cleaned and short CNTs were functionalized with PEG-NH 25000 to obtain a product (sh-MWNTPEG 5000) soluble in aqueous and organic solvents. The method is similar to one used on single wall CNTs synthesized by high pressure catalytic decomposition of carbon monoxide (SWNT, HiPCO $[11,12]$ ). The procedure was optimized for MWNTs synthesized by catalytic chemical vapor deposition (CCVD) at the Polytechnic of the University of Milan. The sh-MWNT-PEG 5000 was used as a filler in a thick, homogeneous and transparent composite (shMWNT-PEG 5000-PMMA) obtained by means of in situ photo-polymerization starting by dispersing the soluble filler in the liquid monomer. The composite was optically, structurally and thermally characterized.

\section{Experimental}

\subsection{Instruments}

\subsubsection{Microwave generator}

In this work we used a SAIREM microwave working at $2.45 \mathrm{GHz}$ and variable power. The principal features of this instrument are: homogeneous distribution of field inside sample holder, capacity of measuring adsorbed power, ease of use. The device has a wave guide with a rectangular section, a cylindrical sample cavity, a black absorber to absorb the power not reflected and not absorbed by the sample. The incident power $(\mathrm{Pi})$, the reflected power $(\mathrm{Pr})$, and the transmitted power (Pt) were continuously monitored by power sensors connected before and after the sample holder. The sample was thermostated by manually regulating the incident power and the temperature of the coolingwater that flowed inside the coil placed close to the external walls around the sample. The temperature of sample was continuously monitored by an optical fiber thermometer (Nortech Reflex-TP21M02) linked to a computer.

\subsubsection{UV-vis spectrometry}

UV-vis absorption spectra were obtained in the range 300-800 nm by using a double-beam Beckman DU-640 spectrophotometer with a resolution of $1.0 \mathrm{~nm}$.

\subsubsection{Infrared spectrometry}

Infrared spectra were collected with solvent compensation in the $900-4000 \mathrm{~cm}^{-1}$ spectral region using a Bruker 
(IFS25) FT-IR spectrometer and a cell equipped with CaF2 windows. The FT-IR spectra of the nanotube (NT) powders were recorded from a pressed disk of the NT mixed with $\mathrm{KBr}$ powder. The pill-composites were placed on the sampler, as prepared. The measurements were carried out at $25^{\circ} \mathrm{C}$ at a spectral resolution of $2 \mathrm{~cm}^{-1}$.

\subsubsection{Nuclear magnetic resonance (NMR) spectroscopy}

${ }^{13} \mathrm{C}$ cross-polarization magic-angle spinning nuclear magnetic resonance $\left({ }^{13} \mathrm{C}\left\{{ }^{1} \mathrm{H}\right\}\right.$ CP-MAS NMR) spectra were obtained at room temperature using a Bruker Avance II $400 \mathrm{MHz}(9,4 \mathrm{~T})$ spectrometer operating at $100.63 \mathrm{MHz}$ for the ${ }^{13} \mathrm{C}$ nucleus with a MAS rate of $13 \mathrm{kHz}$ for 1024 scans, a contact time of $1.5 \mu \mathrm{s}$, and a repetition delay of $2 \mathrm{~s}$. The optimization of the Hartmann-Hahn condition was obtained using an adamantine standard. All samples were placed in a $4 \mathrm{~mm}$ zirconia rotor with KEL-F caps using silica as a filler to avoid inhomogeneities inside the rotor. The proton spin-lattice relaxation time in the rotating frame $\mathrm{T} 1 \rho(\mathrm{H})$ was indirectly determined, with a variable spin-lock (VSL) pulse sequence, by carbon nucleus observation using a $90 \tau$-spin-lock pulse sequence prior to cross-polarization. Data acquisition was performed by ${ }^{1} \mathrm{H}$ decoupling with spin-lock pulse durations ranging from 0.1 to $7.5 \mathrm{~ms}$ and a contact time of $1.5 \mathrm{~ms}$.

\subsubsection{Transmission electron microscopy (TEM)}

TEM micrographs were recorded on a high-resolution transmission electron microscope (HRTEM) JEOL JEM2100 operating at $200 \mathrm{kV}$ accelerating voltage. Elemental analysis was carried out using an Oxford Instruments energy dispersive X-ray detector (EDS). The composite was cut into thin slices using a Leica EM-UC6 ultramicrotome. The $100 \mathrm{~nm}$ slices were deposited on a 200-mesh copper grid, which was carbon-coated to better disperse the high energy electron beam.

\subsubsection{Differential scanning calorimetry (DSC)}

DSC analyses were carried out in a Perkin Elmer DSC7 differential scanning calorimeter under flowing nitrogen atmosphere $\left(20 \mathrm{ml} \mathrm{min}^{-1}\right)$. The instrument was calibrated using the onset temperatures of melting of indium and zinc standards, and the melting enthalpy of indium. Polymer samples (between 5 and $10 \mathrm{mg}$ ) were initially heated from 30 to $200{ }^{\circ} \mathrm{C}$ at $10^{\circ} \mathrm{C} \mathrm{min}{ }^{-1}$, held at that temperature for $1 \mathrm{~min}$ to eliminate thermal history effects, and then cooled to $30^{\circ} \mathrm{C}$ at $10^{\circ} \mathrm{C} \mathrm{min}-1$. They were kept there for $1 \mathrm{~min}$, heated again to $200{ }^{\circ} \mathrm{C}$ at $10^{\circ} \mathrm{C} \mathrm{min}{ }^{-1}$, and cooled to $30^{\circ} \mathrm{C}$ at the same rate.

\subsubsection{Thermogravimetric analysis (TGA)}

TGA analyses were carried out in a Perkin Elmer TGA7 thermogravimetric analyzer. Polymer samples (between 5 and $10 \mathrm{mg}$ ) were heated from 30 to $850{ }^{\circ} \mathrm{C}$ at $20^{\circ} \mathrm{C} \mathrm{min}^{-1}$ under flowing nitrogen $\left(20 \mathrm{ml} \mathrm{min}^{-1}\right)$.

\subsection{Preparation of nanotubes}

The carbon nanotubes were synthesized by a CVD method, at a reaction temperature of $550-750^{\circ} \mathrm{C}$, with an iron catalytic cluster in the laboratory group of Prof.
Mazzocchia, Milano Politecnico University. The NTs appeared as big bundles of micrometer size (TEM micrographies). Several methods were tried to purify and create carboxylate groups on NTs' extremities and defects (cutting). The subsequent steps were etching and functionalization with PEG-NH 5000 (Sigma-Aldrich 672130) to obtain a soluble product to embed in the PMMA matrix.

\subsection{Treatment and purification of nanotubes}

\subsubsection{Cutting ultrasonic assisted method}

During the cutting process the combined action of ultrasound and a sulphonitric mix causes the disaggregation of the bundles and breaking of the nanotubes on the extremities. The fullerenic caps and defects are the most reactive sites because of the presence of pentagons and heptagons in the curvature, consequently they are removed and destroyed. The sulphonitric mix allowed us to obtain a very homogeneous product accompanied by effective exfoliation. The product appeared as a compact film easily removable from the filter.

The cutting process reduces the length of the pristine nanotubes to shorter nanotubes without fullerenic cups (short - sh); the sulfuric acid transfers a proton onto the nitric acid, thus creating, by dehydration, the very reactive nitronium ion. The nitronium ion, assisted by ultrasonic or microwaves, succeeds in reacting with the nanotube defects.

$54 \mathrm{mg}$ of o-MWNT were suspended in $68 \mathrm{ml}$ of a $3: 1$ sulphonitric mixture (H2SO4 (96\%, Aldrich 320501)/ $\mathrm{HNO}_{3}$ (65\%, Aldrich 02650) in a $250 \mathrm{ml}$ flask. This was an extremely exothermic process. The flask was placed for $4 \mathrm{~h}$ in an ultrasonic bath $(45 \mathrm{KHz}, 150 \mathrm{~W})$ at $55^{\circ} \mathrm{C}$. The mixture was diluted $1: 1$ with deionized water to stop the action of acid, centrifuged and separated by supernatant. The solid underwent a second treatment with $16 \mathrm{ml}$ of the sulphonitric mix in the ultrasonic bath for $2 \mathrm{~h}$, diluted $1: 1$ with deionized water, and joined with the supernatant just separated. The mixture was filtered under vacuum on a Millipore $0.1 \mu \mathrm{m}$ VCTP ( $\Phi 47 \mathrm{~mm}$ ) membrane. The filtrate was clear and yellow-colored. The short nanotubes that remained on the filter were washed with $0.1 \mathrm{M} \mathrm{NaOH}$ $(\sim 5 \mathrm{ml})$ until the filtrate no longer assumed a dark brown color (formation of sodium carboxilate). The last acid wash with $\mathrm{HCl} 0.1 \mathrm{M}(\sim 10 \mathrm{ml})$ brings back the extremities into the acid form (colorless filtrate). On the filter remained a black solid that, after drying, became a black film of easily separable purified nanotubes. The yield is $56.5 \%$.

\subsubsection{Cutting microwave assisted method}

Fifty-four milligrams of o-MWNT were suspended in a sulphonitric mixture in a $250 \mathrm{ml}$ cylindrical container, and irradiated in the microwave holder under several combinations of time and power, using pauses to avoid excessive heating in the environment: $A 1:(5+5)$ minutes at $200 \mathrm{~W}$; B1: $5 \mathrm{~min}$. at $50 \mathrm{~W}+5 \mathrm{~min}$. at $100 \mathrm{~W}+5 \mathrm{~min}$. at $150 \mathrm{~W}$; B2: etched MWNT; F2: $(3.5+3.5) \mathrm{min}$. at $300 \mathrm{~W}$; D2: $(5+5)$ min. at $300 \mathrm{~W}$. After diluting the mixture with deionized water, it was filtered under vacuum on a Millipore membrane $0.1 \mu \mathrm{m}$ VCTP ( $\Phi 47 \mathrm{~mm}$, Sigma-Aldrich). The solid on the filter was washed with $0.1 \mathrm{M} \mathrm{NaOH}$ and 
with $0.1 \mathrm{M} \mathrm{HCl}$, until the filtrate was clear. On the filter remained a black compact solid of shortened nanotubes. ("+" means a pause among the treatments to avoid the excessive heating of mix and consequent sample degradation).

\subsubsection{Cutting $\mathrm{HNO}_{3}$ refluxing [13]}

Fifty-four milligrams of o-MWNT were suspended in concentrated nitric acid (65\%, Aldrich). The suspension was refluxed for $24 \mathrm{~h}$. After diluting the mixture with deionized water, it was filtered under vacuum on a Millipore membrane $0.1 \mu \mathrm{m}$ VCTP ( $\Phi 47 \mathrm{~mm}$, Sigma-Aldrich). The solid on the filter was washed with $0.1 \mathrm{M} \mathrm{NaOH}$ and with $0.1 \mathrm{M} \mathrm{HCl}$, until the filtrate was clear. On the filter remained a black frayed solid of shortened nanotubes. The yield was $44.2 \%$.

\subsubsection{Etching}

The etching process, through a strongly oxidative mixture called "Piranha" [14], eliminates the carbon impurities formed in the cutting process, and completely oxidizes the NT extremities to carboxylic residues. The cutting process produced a large amount of amorphous carbon trapped inside the bundles of short nanotubes. To purify these carbon impurities and to create carboxylic sites, the nanotubes were dipped in a strong oxidizing solution, a $20 \mathrm{ml}$ "Piranha" solution $\left(4: 1 \mathrm{H}_{2} \mathrm{SO}_{4}(96 \%) / \mathrm{H}_{2} \mathrm{O}_{2}\right.$ (30\%) 4:1), under stirring, for $30 \mathrm{~min}$ at a temperature of $70^{\circ} \mathrm{C}$. This was a very exothermic process. After cooling to room temperature the solution was diluted 1:1 with deionized water, and filtered under vacuum on a Millipore membrane $0.1 \mu \mathrm{m}$ VCTP $(\Phi 47 \mathrm{~mm}$ ). The solid was washed with $0.1 \mathrm{M} \mathrm{NaOH}(\sim 5 \mathrm{ml})$ until comparing brown filtrate, and with $0.1 \mathrm{M} \mathrm{HCl}(\sim 10 \mathrm{ml})$ until the filtrate was clear. The filter was dried under an IR lamp separating the product as a smooth black film.

\subsubsection{Functionalization with polyethylene glycol (PEG)}

After the first two processes the shortened and cleaned nanotubes showed carboxylic extremities that could undergo condensation and bind functional groups such as amines or alcohols to give amides or esters. However, an intermediate step, acyl chloride formation, was necessary for this reaction to occur.

Seventeen milligrams of oxidized sh-MWNT-COOH were dispersed in a $25 \mathrm{ml}$ flask with bubble cooling, under stirring, in an excess of $\mathrm{SOCl}_{2}\left(7 \mathrm{ml}, 8 \times 10^{-2} \mathrm{~mol}\right)$ containing $5 \%$ in volume of anhydrous dimethylformamide (DMF). The dispersion was maintained at $70^{\circ} \mathrm{C}$ under stirring for $24 \mathrm{~h}$, in nitrogen atmosphere; a yellowing and exfoliation of $\mathrm{SOCl}_{2}$ was observable, with the volume of black solid increasing. The mixture was separated by centrifugation, followed by decantation. After removing the supernatant fluid, the solid was washed with anhydrous toluene until clear supernatant. The black solid was very sensitive to air humidity, and so it was quickly dried under vacuum and temporarily stored under flowing nitrogen.

Acyl chloride can condense with amine: $31.5 \mathrm{mg}$ of shMWNTCOCl and $300 \mathrm{mg}$ of polyethylene glycol-amine (PEG-NH $\mathrm{NH}_{2}, 5000$ dalton) were mixed in a $25 \mathrm{ml}$ flask under stirring. The mixture was heated at $90{ }^{\circ} \mathrm{C}$ and maintained at this temperature for $96 \mathrm{~h}$ in nitrogen atmosphere and
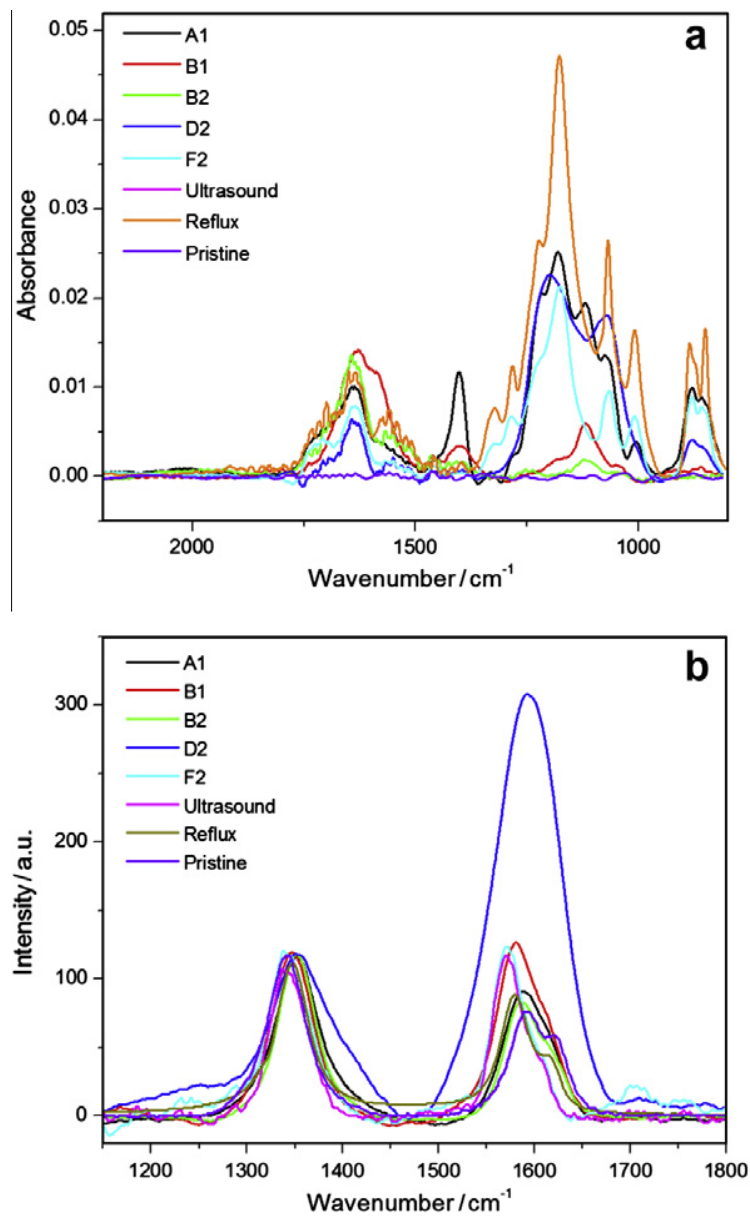

Fig. 1. (a) IR spectra in the range $800-2000 \mathrm{~cm}^{-1}$ and (b) Raman spectra in the range $1200-1800 \mathrm{~cm}^{-1}$, of sh-MWNT after different cutting microwave assisted treatments.

with moderate stirring. The mixture, after cooling to room temperature, was dissolved in $10 \mathrm{ml}$ of deionized warm $\mathrm{H}_{2} \mathrm{O}\left(\sim 50^{\circ} \mathrm{C}\right)$ and was placed in an ultrasonic bath to completely remove the solid from the flask. The aqueous mixture was filtered under vacuum on a Millipore membrane $0.1 \mu \mathrm{m}$ VCTP ( $\Phi 47 \mathrm{~mm}$ ), until obtaining a clear filtrate. The black solid was washed several times with deionized water to avoid drying. The presence of PEG in the filtrate was monitored by thin-layer-chromatography (eluent:

Table 1

Yield, intensity ratios of G and D bands, and IR peaks for each purification.

\begin{tabular}{llll}
\hline Sample & $\begin{array}{l}\text { Total } \\
\text { yield }(\%)\end{array}$ & $\begin{array}{l}\text { Raman } \\
\text { IG/ID }\end{array}$ & IR peaks $\left(\mathrm{cm}^{-1}\right)$ \\
\hline $\mathrm{A} 1$ & 66.1 & $<1$ & $\begin{array}{l}1634(\mathrm{~m}), 1401(\mathrm{~s}), 1180(\mathrm{~m}), \\
1065(\mathrm{~m})\end{array}$ \\
$\mathrm{B} 1$ & 70.1 & 1.06 & $1634(\mathrm{~s}), 1585(\mathrm{~s}), 1401(\mathrm{w})$ \\
$\mathrm{B} 2$ & 99.0 & $<1$ & $1634(\mathrm{~s})$ \\
$\mathrm{D} 2$ & 16.7 & 2.61 & $1634(\mathrm{~m}), 1180(\mathrm{~m}), 1065(\mathrm{~s})$ \\
$\mathrm{F} 2$ & 10.3 & 1.04 & $1634(\mathrm{~s}), 1180(\mathrm{~m}), 1065(\mathrm{w})$ \\
Ultrasonic & 56.5 & 1.03 & $1634(\mathrm{~s}), 1180(\mathrm{~s}), 1065(\mathrm{~s})$ \\
$\mathrm{HNO}_{3}$ & 44.2 & $<1$ & $1634(\mathrm{w}), 1180(\mathrm{w})$ \\
$\quad$ reflux & & & \\
\hline
\end{tabular}



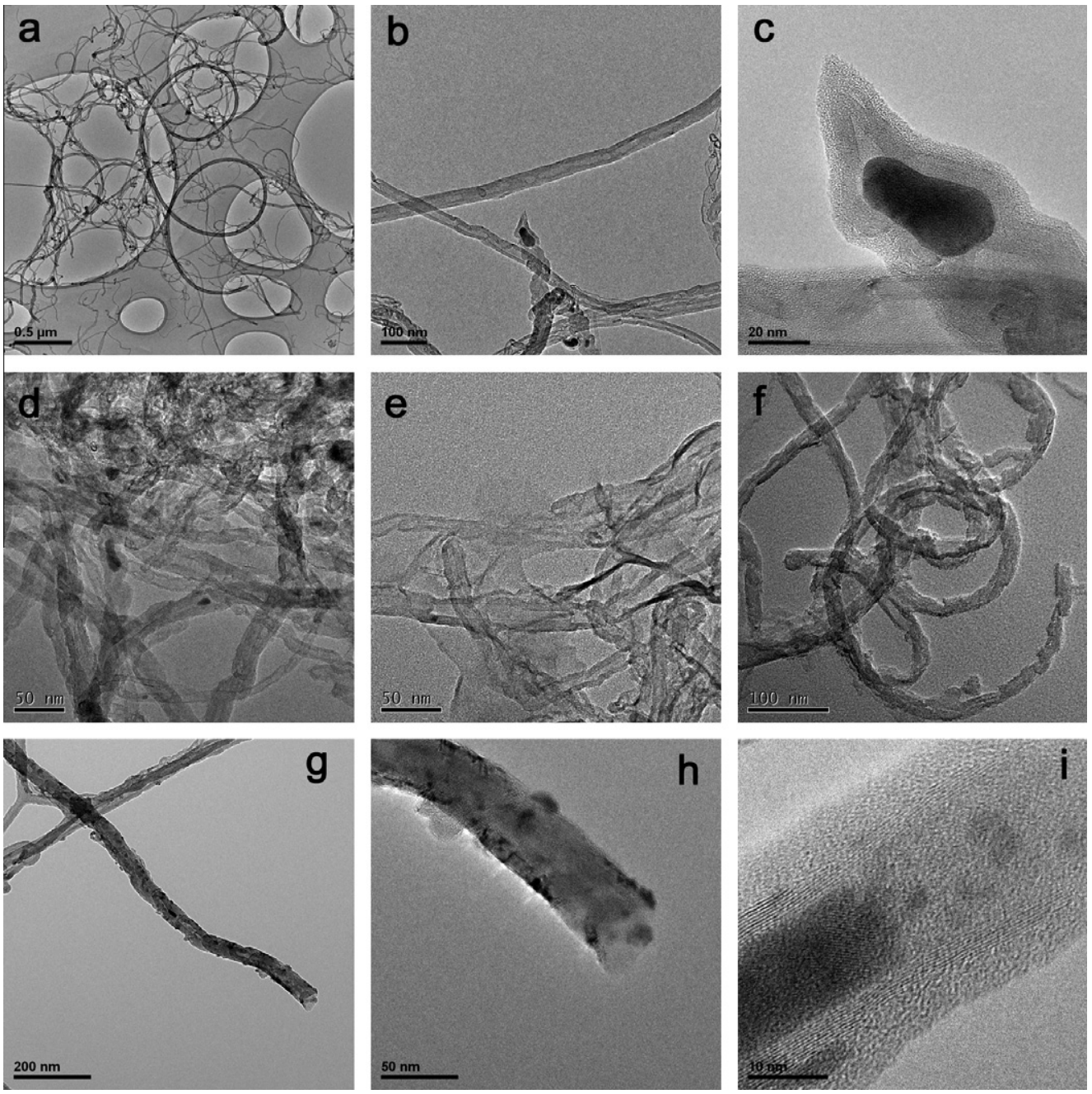

Fig. 2. TEM micrographs of (a) pristine MWNT, (b) carbon impurities and (c) metal synthesis catalysts, as well as (d) B1, (e) ultrasonic, and (f) HNO ${ }_{3}$ reflux treated samples; TEM micrographs at different enlargements of ( $g$ ) sh-MWNT-PEG, (h) without fullerenic caps and (i) with PEG-NH ${ }_{2}$ outside and inside the nanotube.

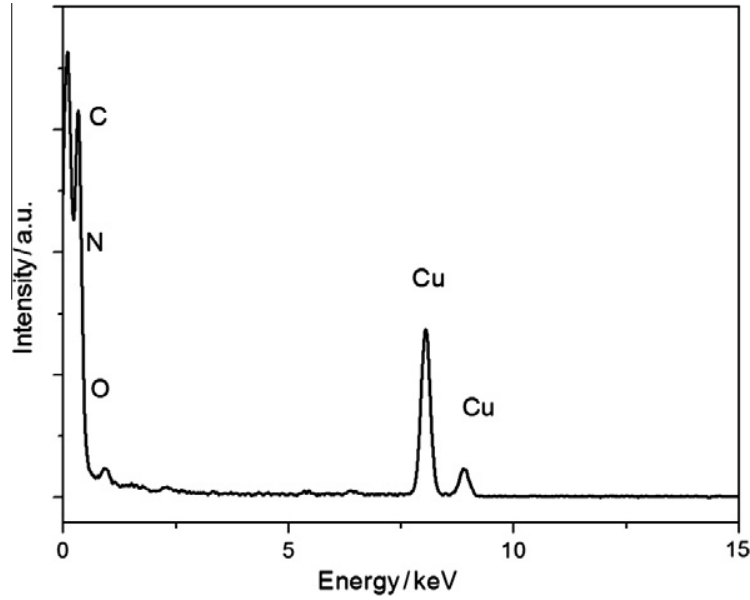

Fig. 3. EDS analysis of sh-MWNT-PEG. chloroform/ethanol 8:2, $R f=0.6$ after dipping with $\mathrm{KMnO}_{4}$ ). The solid on the filter was washed with $\sim 15 \mathrm{ml}$ of ethanol and $\sim 15 \mathrm{ml}$ of methanol without drying. The filtration was very slow. The black solid, still wet, was washed with tetrahydrofuran (THF) and separated by centrifugation ( $5 \mathrm{ml}, 10 \mathrm{~min}$ of sonication, centrifugation), obtaining a black supernatant. Sonication, followed by centrifugation, was repeatedly performed until a clear supernatant was obtained. The supernatants $(150 \mathrm{ml})$ were mixed in a $250 \mathrm{ml}$ flask and filtered under vacuum by a Millipore membrane $0.5 \mu \mathrm{m}$ PTFE ( $\Phi: 47 \mathrm{~mm}$ ). The clear black solution was concentrated and the product precipitated by cyclohexane. It was washed with the same solvent, then with diethylether, and then dried under vacuum. The final yield was $50 \%$ in weight.

\subsection{Composite preparation}

The sh-MWNT-PEG-PMMA composites (1\%) were obtained by adding sh-MWNT-PEG into a glass vial contain- 


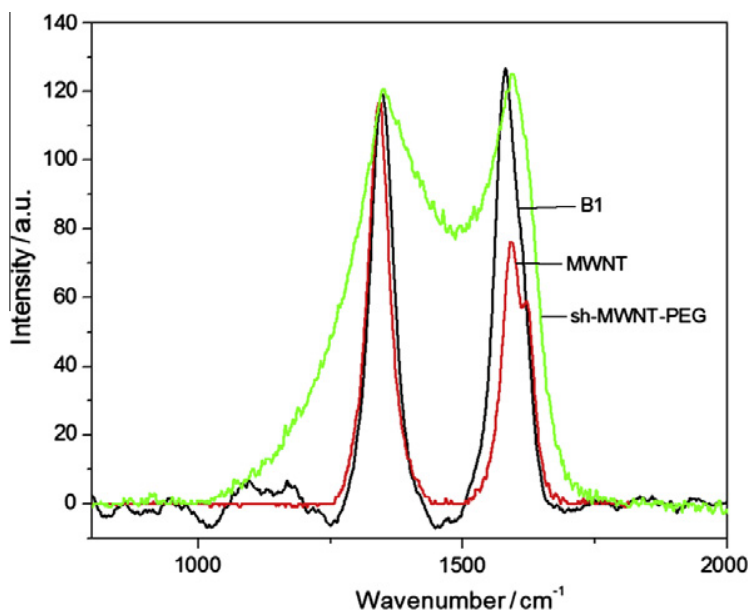

Fig. 4. Raman spectra of pristine CNT, B1 and sh-MWNT-PEG.

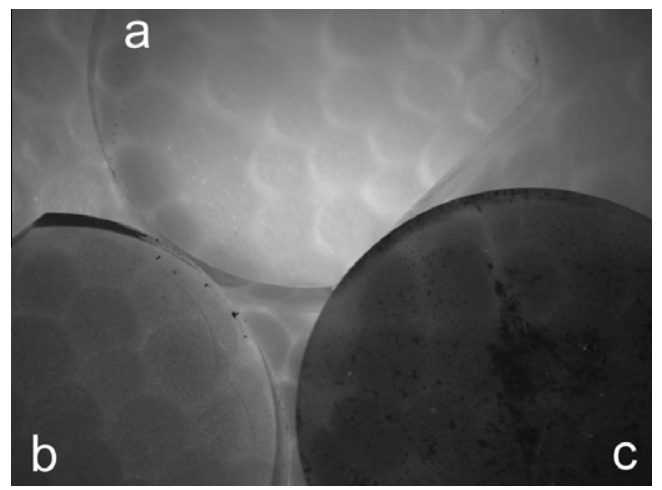

Fig. 5. Optical microscopy picture of (a) PMMA, (b) sh-MWNT-PEGPMMA and (c) MWNT-PMMA.

ing $5 \mathrm{ml}$ of methylmetacrylate (MMA). The mixture was placed inside a ultrasonic bath for one hour to facilitate

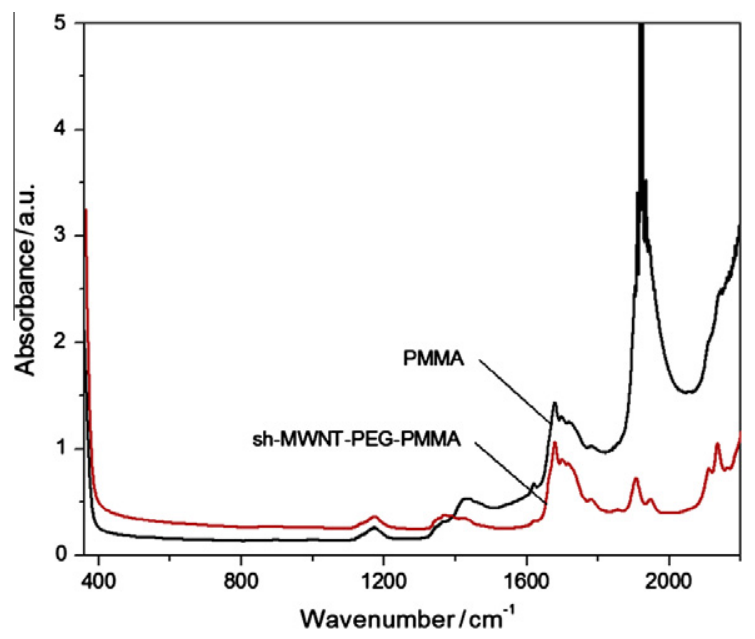

Fig. 6. UV-vis-NIR spectra of pure PMMA and the sh-MWNT-PEG-PMMA composite.

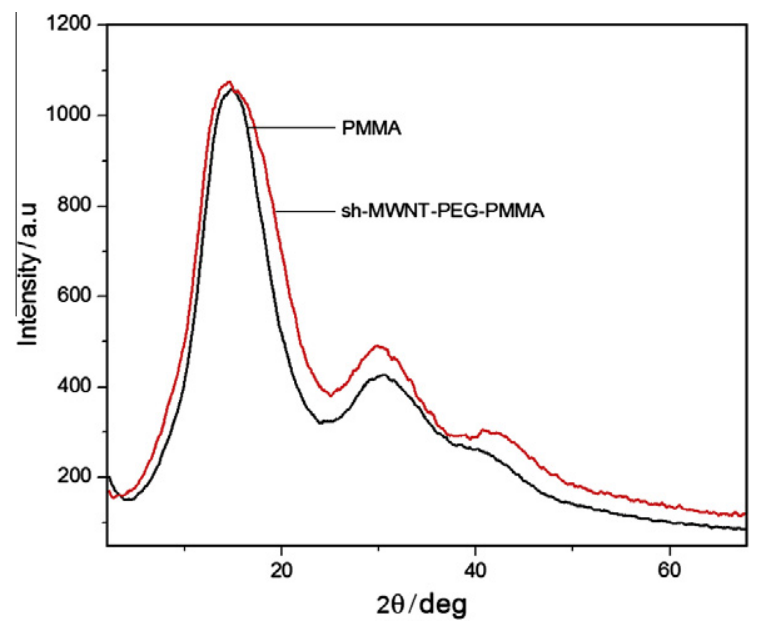

Fig. 7. XRD diffractograms of PMMA and the sh-MWNT-PEG-PMMA composite.

the particles' dispersion. The radical initiator 2,2-dietossiacetofenone was added to start the process of photo-polymerization. The dispersion was continuously stirred and irradiated by a UV lamp ( $256 \mathrm{~nm}, 17 \mathrm{~W}$ ) until the end of the polymerization. The composite was cut in round tablets with thickness $2.5 \mathrm{~mm}$ and diameter $10 \mathrm{~mm}$. A composite containing $1 \%$ pristine nanotubes was prepared for comparison.

\section{Results and discussion}

The cutting methods were monitored by FT-IR and RAMAN spectroscopy. The IR spectroscopy works as an indicator of new bond formation. The acid-oxidative attack produces several functional groups on the NT extremities. Raman spectroscopy allows the observation of the chemical modification produced on the MWNT by oxidative cutting, as well as information on the purity and the presence of defects on the walls. This information is obtained from the $G$ and $D$ bands. The G-band is the sum of two frequencies: the first one $\left(1572 \mathrm{~cm}^{-1}\right)$ is attributed to bond deformations along the nanotube and the other one $\left(1600 \mathrm{~cm}^{-1}\right)$ is attributed to stretching of the $\mathrm{C}-\mathrm{C}$ bonds, not parallel to the axis. The D-band is called the "disorder band" and is found between 1250 and $1450 \mathrm{~cm}^{-1}$. The defects of the nanotubes, for example the random formation of pentagon-heptagon couples, are characterized by this "vibrational line". The presence of amorphous carbon is related to the band intensity.

The IR spectra (Fig. 1) of the samples treated according to the B1, B2 and ultrasound methods have a strong absorption band centered at $1634 \mathrm{~cm}^{-1}$ attributed to the stretching $\mathrm{v}(\mathrm{C}=\mathrm{C})$ and the presence of water. The spectrum of the B1 sample shows a big shoulder at $1585 \mathrm{~cm}^{-1}$ attributed to the stretching $\mathrm{v}(\mathrm{C}=\mathrm{C})$ and $(\mathrm{O}-\mathrm{H})$ vibrational deformation. The spectrum of the $\mathrm{A} 1$ sample presents the most intense absorption at $1415 \mathrm{~cm}^{-1}$ (C) attributed to the symmetric stretching of the carboxylic groups [15]. The sample treated by the ultrasound method shows the strongest absorptions at 1714, 1178 and 1065 attributed to the 


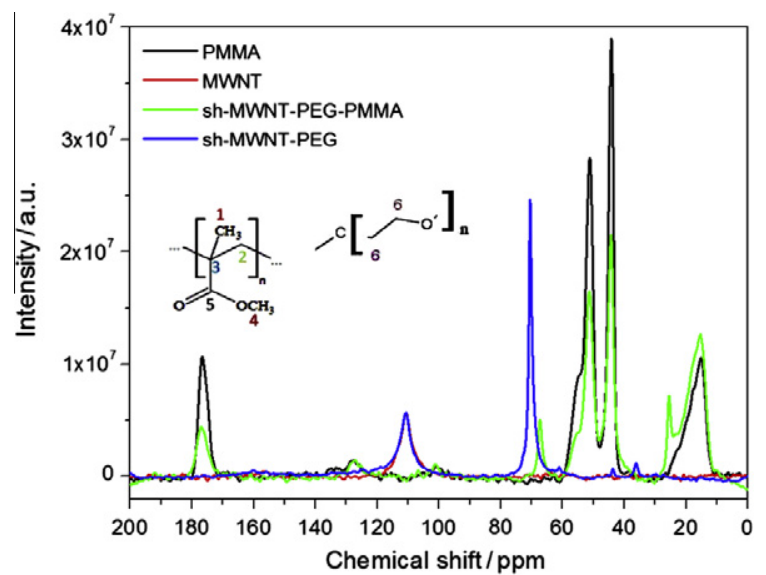

Fig. 8. Comparison of the NMR HPDec spectra of MWNT, sh-MWNT-PEG and the sh-MWNT-PEG-PMMA composite.

Table 2

T1 $\rho(H)$ values of PMMA and the MWNT-PMMA composite.

\begin{tabular}{llll}
\hline & PMMA & MWNT-PMMA & sh-MWNT-PEG-PMMA \\
\hline Peak/ppm & T1 $\rho(\mathrm{H}) / \mathrm{ms}$ & T1 $\rho(\mathrm{H}) / \mathrm{ms}$ & $\mathrm{T} 1 \rho(\mathrm{H}) / \mathrm{ms}$ \\
$16.4(1)$ & $6.6 \pm 0.2$ & $23.5 \pm 0.5$ & $10.9 \pm 0.5$ \\
$45.5(2)$ & $7.0 \pm 0.2$ & $21.4 \pm 0.3$ & $6.5 \pm 0.3$ \\
$52.7(3)$ & $7.6 \pm 0.3$ & $20.7 \pm 0.2$ & $7.8 \pm 0.4$ \\
$55.1(4)$ & $7.2 \pm 0.2$ & $18.0 \pm 0.4$ & $9.9 \pm 0.5$ \\
$178.1(5)$ & $6.4 \pm 0.4$ & $27.3 \pm 0.7$ & $12.5 \pm 0.6$ \\
\hline
\end{tabular}

$v(C=O), v(C-O)$ and $v(C-O-C)$, which is a sign of stronger oxidation. The same, but weaker absorptions, are present in the A1, D2 and F2 samples.

The Raman spectra in Fig. 1 show that, after normalizing the spectra with respect to the D band, the intensity of the $\mathrm{G}$-band increases with increasing treatment times. The B1 and F2 samples show the same intensity of longitudinal stretching, but the B1 sample has a stronger intensity of stretching not parallel to the axis. To avoid any misunderstanding, a pristine nanotube sample was dispersed in water exposed to MW for $35 \mathrm{~min}$ at $250 \mathrm{~W}$. There was no cutting and the cap breaking off did not occur in water.

The quality of treatment was evaluated considering not only spectroscopy data but also the yield of sh-MWNT. The best quality of Raman data (D2, ratio of G/D) corresponds to a very low yield. The $\mathrm{HNO}_{3}$ treatment drastically reduced the yield and increased the amorphous carbon impurities, thus decreasing the IG/ID ratio, which indicates CNT damage.

Table 1 summarizes the values of synthesis yield, ratio of $G$ and $D$ bands, and IR peaks of the purified products.

A TEM study of the products of the different purifications was carried out to understand what changes occurred in the nanotubes' walls and structures (Fig. 2). The pristine nanotubes appear entangled in bundles, and they have a uniform diameter and micrometric length (a), with metallic clusters from the growth catalyst inside (b), and some amorphous carbon on the walls (c). All the treatments (d-f) show the disappearance of the metal clusters and the carbon, and only the $\mathrm{HNO}_{3}$ reflux treatment gave rise to degradation (f). Transmission electron microscopy interfaced with EDS gave us information about nanotube sizes and their extent of purification from amorphous carbon and metal clusters. The sh-MWNT-PEG are shorter, without fullerenic caps or other carbon impurities and no obvious synthesis catalyst (Fig. 2g), and the PEG appears to adhere to the nanotube walls (Fig. 2h). EDS supplies and confirms the information from the elemental analysis on the composition: the presence of carbon, oxygen and nitrogen are obvious (Fig. 3). During the TEM analysis it was interesting to observe the presence of crystalline material inside the nanotube (Fig. 2i), which probably was PEG$\mathrm{NH}_{2}$.

The sh-MWNT-PEG product was characterized by several spectroscopic techniques. The Raman spectra are shown in Fig. 4. Comparison of the spectra of pristine MWNT and sh-MWNT-PEG confirms the relative decrease of structural disorder, defects, and amorphous carbon associated with the D band. The sh-MWNT (B1) did not show any improvement, and the change in spectrum shape is due to the weak response of NT hidden by the big amount of PEG.

After characterization of the different untreated and treated MWNTs, sh-MWNT-PEG and pristine MWNT were embedded in PMMA, creating the composites sh-MWNT-
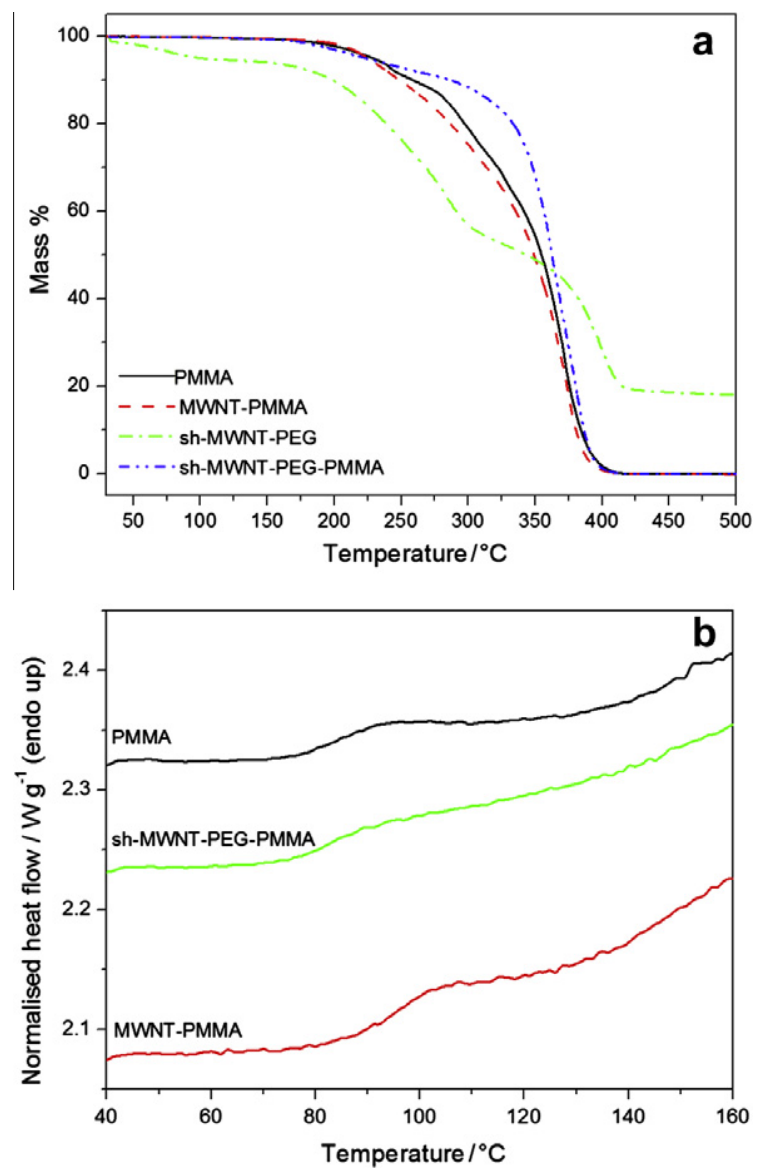

Fig. 9. (a) TGA and (b) DSC curves of PMMA, sh-MWNT-PEG, and the different composites. 
Table 3

Glass transition temperatures and transition $\Delta C \mathrm{p}$ values of the composites.

\begin{tabular}{lll}
\hline & $T_{\mathrm{g}}\left({ }^{\circ} \mathrm{C}\right)$ & $\Delta C_{\mathrm{p}}\left(\mathrm{J} \mathrm{g}^{-1}{ }^{\circ} \mathrm{C}^{-1}\right)$ \\
\hline PMMA & 84 & 0.2 \\
MWNT-PMMA & 93 & 0.3 \\
Sh-MWNT-PEG-PMMA & 84 & 0.2 \\
\hline
\end{tabular}

PEG-PMMA and MWNT-PMMA. Optical comparison of the two composites (Fig. 5) shows that the sh-MWNT-PEGPMMA was more homogeneous and transparent than MWNT-PMMA. The pristine MWNT was inclined to agglomerate and so create macroscopic bundles.

The sh-MWNT-PEG-PMMA was characterized by UV-vis spectroscopy, XRD, ${ }^{13} \mathrm{C}-\mathrm{NMR}$ CPMAS, HPDEC, high resolution TEM, TGA, and DSC. The UV-vis-NIR spectra of pure PMMA and the sh-MWNT-PEG-PMMA composite are shown in Fig. 6. The sh-MWNT-PEG-PMMA composite absorbs between 400 and $1300 \mathrm{~nm}$; the presence of characteristic peaks around $1340-1400 \mathrm{~nm}$ indicates the presence of NT; the absorption of the composite is less intense than that of pure PMMA between 1400 and $2200 \mathrm{~nm}$. This is uncommon behavior, but MWNT is probably transparent in the IR wavenumber range and consequently the absorption of the nanocomposite is less intense than that of PMMA.

Fig. 7 shows the XRD spectra of PMMA and the shMWNT-PEG-PMMA composite. The peak at $2 \theta=14.5^{\circ}$ broadens for the composites. It also appears as the sum of more than one contribution and deconvolution provides two peaks at $14.4^{\circ}$ and $15.8^{\circ}$. The third peak, centered at $42.3^{\circ}$, appears, in the composite, more intense than the one for pure PMMA. The filler undoubtedly induces some small structural differences into the matrix.

The ${ }^{13} \mathrm{C}$ NMR High Power Decoupling (HPDec) spectrum (Fig. 8) shows no visible change in the chemical shift of the carbon nanotubes before and after the functionalization, although the peak is broadened because of the large number of carbons in each nanotube. The peak at $112 \mathrm{ppm}$ is characteristic of graphitic systems and the one at $70 \mathrm{ppm}$ is the methylenic peak of PEG. A 4 ppm shift of the ethylene glycol carbon peak towards a higher field means the nuclei became more shielded after composite formation. An interaction exists between electron donors of PMMA and the methylenic groups of PEG bonded to the nanotubes.

The relaxation spin-lattice data $(\mathrm{T} 1 \rho)$, reported in Table 2 , show an increase in relaxation times T1 $\rho$ of all the carbons with respect to that of PMMA. This means that the polymeric chain stiffened uniformly after MWNT embedding. A possible explanation can be the longitudinal displacement of nanotubes along the polymeric chain axis. The plasticity of PEG uniformly lowers the values of $\mathrm{T} 1 \rho(\mathrm{H})$, which indicates weak interactions of the filler with carbons 1,4 and 5 .

Thermogravimetric analysis was carried out on the material to evaluate possible interactions between the filler and the polymer chains. The TGA curves of PMMA, shMWNT-PEG and the composites are reported in Fig. 8. Interaction with the MWNT either immobilizes the polymer chains and free radical chains, which should retard the degradation process, or slows down the diffusion of the degradation products out of the sample, or both. The introduction of MWNT does not significantly improve the thermal stability of the matrix. The most significant improvement is seen in the case of sh-MWNT-PEG, probably due to better dispersion of the filler.

The DSC data show an increase in glass transition temperature $\left(T_{\mathrm{g}}\right)$ of the MWNT-PMMA nanocomposite compared to that of the pure matrix (Fig. 9, Table 3). It seems as if, at this content, there was an appreciable interaction between the nanotubes and the polymer chains (even in the absence of PEG functionalization), which reduced the mobility of the polymer chains and increased its $T_{\mathrm{g}}$. The presence of PEG functionalization actually seems to plasticize the PMMA which gives rise to a $T_{\mathrm{g}}$ similar to that of pure PMMA.

\section{Conclusions}

A new fast and clean method based on microwave radiation was used for the purification of MWNT before introducing the nanotubes into PMMA during in situ polymerization. The pristine nanotubes had many defects, so the acid attack had to be calibrated to obtain a satisfying yield. The nanotubes were shortened, and these purified nanotubes were functionalized with polyethylene glycol. This functionalization was important for the formation of a transparent and homogeneous composite. The latter was transparent over the whole visible spectrum. The embedding of sh-MWNT-PEG into PMMA did not cause substantial structural change, and the nanotubes were arranged longitudinal along the polymeric chain axes. The polyethylene glycol interacted with the PMMA, causing the MWNT to reduce the mobility of the polymeric chains, and influencing the thermal properties.

\section{Acknowledgements}

Antonio Zanotto acknowledges the MIUR and INSTM fellowship for his work on "TEM analysis on nanostructured composites", and Prof. Mazzocchia, Milano Politecnico University for the carbon nanotubes. NMR and TEM data were provided by Centro Grandi Apparecchiature Uninetlab, Univerità di Palermo. The National Research Foundation in South Africa and the University of the Free State are acknowledged for financial support.

\section{References}

[1] Spitalskya Z, Tasisb D, Papagelis K, Galiotis C. Prog Polym Sci 2010;35:357-401.

[2] MacKenzie K, Dunens O, Harris AT. Sep Purif Technol 2009;66:209-22.

[3] Zujin S, Yongfu L, Fuhui L, Xihuang Z, Zhennan G, Yaogang Z, Iijima Sumio. Solid State Commun 1999;112:35-7.

[4] Chen J, Hamon MA, Hu H, Chen YS, Rao AM, Eklund PC, et al. Science 1998;282:95-8.

[5] Hu H, Zhao B, Itkis ME, Haddon RC. J Phys Chem B 2003;107:13838-42.

[6] Wadhawan A, Garrett D, Perez JM. Appl Phys Lett 2003;83:2683-5.

[7] Imholt TJ, Dyke CA, Hasslacher B, Perez JM, Price DW, Roberts JA, et al. Chem Mater 2003;15:3969-70.

[8] Frank S, Poncharal P, Wang ZL, DeHeer WA. Science 1998;280:1744-6. 
[9] Chen Y, Iqbal Z, Mitra S. Adv Funct Mater 2007;17:3946-51.

[10] Schmidt RH, Kinloch IA, Burgess AN, Windle AH. Langmuir 2007;23:5707.

[11] Menna E, Scorrano G, Maggini M, Cavallaro M, Della Negra F, Bozio R, et al. Arkivoc 2003;12:64-73.

[12] Della Negra F, Meneghetti M, Menna E. Fullerene, Nanotubes, Carbon Nanostruct 2003;11:25-34.
[13] Qin Y, Liu L, Shi J, Wu W, Zhang J, Guo ZX. Chem Mater 2003:15:3256-60

[14] Liu J, Rinzler AG, Dai HJ, Hafner JH, Bradley RK, Boul PJ, et al. Science 1998;280:1253-6.

[15] Yan X-B, Tay BK, Yang Y. J Phys Chem B 2006;110:25844-9. 